\section{Computer-Based Order Entry Decreases Duration of Indwelling Urinary Catheterization in Hospitalized Patients}

Paul B. Cornia, MD, John K. Amory, MD, Shelagh Fraser, MD, Sanjay Saint, MD, MPH, Benjamin A. Lipsky, MD

$\mathrm{U}$ $\mathrm{p}$ to $25 \%$ of hospitalized patients will have an indwelling urinary catheter inserted (1). While most of these catheters are required for optimal patient care, about one in five is unnecessary (2). Catheter-related urinary tract infections are the leading cause of nosocomial infection. They account for up to $40 \%$ of hospital-acquired infections, with an incidence of $3 \%$ to $10 \%$ per day of indwelling catheterization (1,3-5). Bacteremia occurs in $1 \%$ to $4 \%$ of those who develop nosocomial bacteriuria (6). Most patients also find an indwelling urinary catheter to be uncomfortable and activity restricting (7). Given these potential morbidities, it is remarkable how infrequently the use of a urinary catheter is documented by a physician's order in the medical record (8).

The strongest predictor for catheter-associated bacteriuria is the duration of catheterization $(3,4)$. Thus, methods to shorten this period should reduce the risk of nosocomial urinary tract infection, yet physicians are often unaware that their own hospitalized patients have a urinary catheter in place (9). Furthermore, these "forgotten" catheters were about twice as likely to be unnecessary as those remembered by the physician (9). Thus, a system that would remind physicians which of their patients had

From the Primary and Specialty Medicine Service (PBC, JKA, SF, BAL), Veterans Affairs Puget Sound Health Care System, and the Department of Medicine, University of Washington School of Medicine, Seattle, Washington; and the Ann Arbor VA Medical Center (SS), and Department of Internal Medicine and Patient Safety Enhancement Program, University of Michigan Health System, Ann Arbor, Michigan.

This project was supported by a grant from the Research Foundation for the Prevention of Complications Associated with Health Care, Washington D.C., and by the Department of Veterans Affairs. Dr. Saint is supported by a Career Development Award from the Health Services Research \& Development Program of the Department of Veterans Affairs and a Patient Safety Developmental Center grant (P20-HS11540) from the Agency for Healthcare Research and Quality, Rockville, Maryland.

Requests for reprints should be addressed to Paul B. Cornia, MD, General Internal Medicine Clinic, S-111, 1660 South Columbian Way, Seattle, Washington 98108, or paul.cornia@med.va.gov.

Manuscript submitted March 12, 2002, and accepted in revised form October 28, 2002. urinary catheters might shorten the duration of catheterization.

One such approach involves using automatic reminders to physicians through computerized medical record systems (10-15). At our Veterans Affairs (VA) medical center, nearly all patient records and orders are entered into a computer. We hypothesized that a computer-based order for inserting an indwelling urinary catheter, combined with computer-generated reminders to remove the catheter, would improve documentation of urinary catheters, alert physicians that the catheter was in place, and encourage discontinuing catheterization that was no longer required.

\section{METHODS}

All inpatients at the VA Puget Sound Health Care System are cared for by teams of resident physicians supervised by University of Washington faculty. Patients on the medicine and cardiology services are predominantly housed on two hospital wards (second and fourth floor), with most of each team's patients on a single ward. During the first 8 weeks of the study, the fourth floor served as the study ward and the second floor served as the control ward; during the second 8 weeks, the second floor became the study ward and the fourth floor became the control.

At the beginning of each month, a training session is held to teach incoming residents about the computer system. During this session, one of the investigators (PBC) explained the purpose of the study, including the direct association between duration of catheterization and catheter-associated urinary tract infection, and instructed residents on use of the computerized urinary catheter study order. We also explained the purpose of the study to the ward nurses and encouraged them to remind physicians to place a computerized order for each indwelling urinary catheter. Physicians whose patients were located on the study ward had three options when ordering an indwelling urinary catheter: using the designated computer study order (Figure), entering a standard written order, or not entering an order. The computer study order was not available for patients located on the control ward.

The computer study order also required that an indication be selected for its placement (e.g., acute urinary retention), provided routine catheter care instructions, and noted a default stop date of 72 hours after placement. The computer-generated reminder that appeared 72 hours after placement asked the ordering physician to either renew or discontinue the catheter order. No computer-generated reminders were delivered for standard written orders.

All patients admitted to the medicine and cardiology services between November 15, 2000, and March 6, 2001, were followed prospectively. Each morning, the investigator 


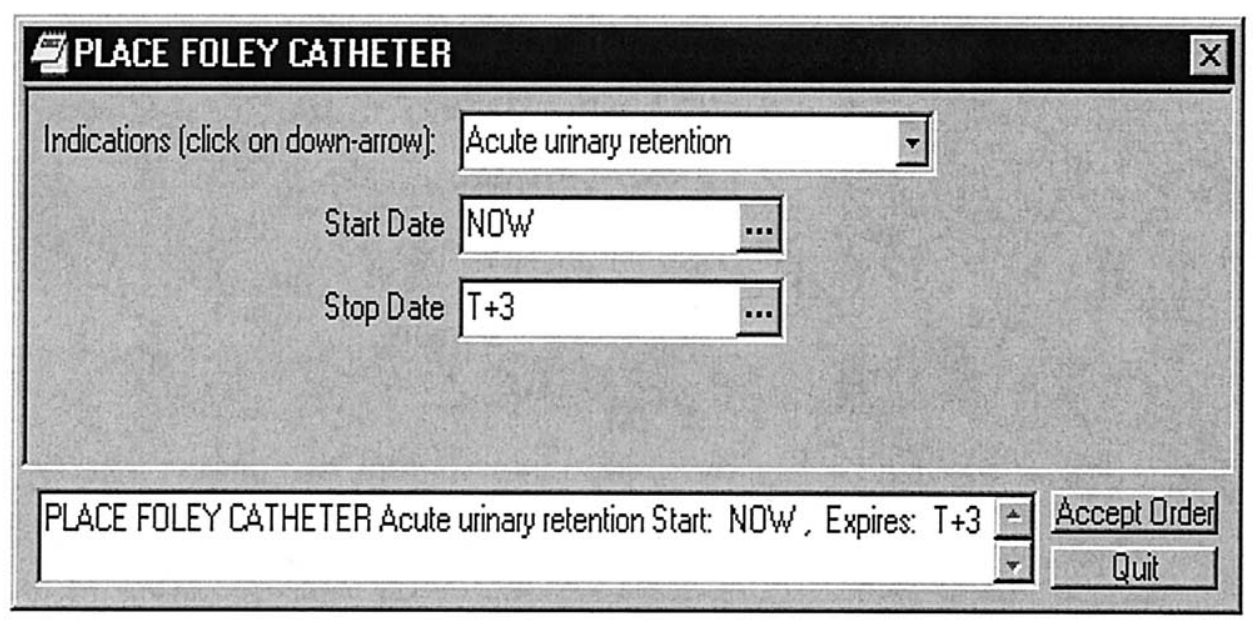

Figure. Order for inserting an indwelling urinary catheter from the computerized patient record system.

checked all patients on the study and control wards for the presence of an indwelling urinary catheter. Patients who had a newly inserted urinary catheter were included in the study. We reviewed the chart to see if there was an order to place the catheter, to determine when the catheter was inserted, and followed patients daily for the duration of their hospitalization. Patients who were transferred to a nonmedicine or noncardiology service, or to a ward other than the second or fourth floor, were removed from the study.

The primary study outcome was the number of days of urethral catheterization per patient with a catheter. The occurrence of catheter-associated urinary tract infection, defined by growth from a urine specimen aseptically aspirated from the catheter (after the first day of catheterization) of $\geq 100$ colony-forming units of a predominant pathogen (16) or $\geq 10$ leukocytes per high-power field on urinalysis in a patient with a clinical diagnosis of a urinary tract infection, was also noted. Urine specimens for culture were sent at the discretion of the physician caring for the patient.

The study was approved by the Human Subjects Committee of the University of Washington and the Research and Development Committee of the VA Puget Sound Health Care System. Written informed consent was not required from patients or providers as the standard of care was to not use computerized urinary catheter orders.

\section{Statistical Analysis}

The statistical significance of differences in days of catheterization between groups was determined with an unpaired $t$ test. Proportions were compared using the Fisher exact test. For all comparisons, a $P$ value $<0.05$ (twosided) was considered statistically significant.

\section{RESULTS}

There were 742 patients admitted to the medicine and cardiology services during the study; 94 patients were ex- cluded following transfer to a ward or treating specialty not included in the study. There was no statistically significant difference between the rates of indwelling urinary catheter use between study wards $(12 \%$ [36/311]) and control wards ( $10 \%$ [34/337]; $P=0.15)$. Of the 36 patients who were on a study ward when the catheter was placed, 33 (92\%) had a charted order. Of the 34 patients on a control ward who had a catheter inserted, only 10 (29\%) had the order documented $(P<0.001$, compared with the study ward; Table 1).

The mean duration of catheterization was 3 days shorter (5 days vs. 8 days) on the study ward $(P=0.03$; Table 2$)$. During the study period, eight catheter-associated urinary tract infections were diagnosed; five in patients on a study ward and three in patients on a control ward $(P=0.71$; Table 2). Of the 5 patients who required reinsertion of a urinary catheter, only 1 had received an automatic computer order to remove the previous catheter.

\section{DISCUSSION}

Indwelling urinary catheterization is a common practice in hospitalized patients. Because these devices cause patient discomfort and are responsible for substantial morbidity (17), they have been called a "one-point

Table 1. Documentation in Medical Record That an Indwelling Urinary Catheter Was Placed

\begin{tabular}{lccc}
\hline & Control Ward & Study Ward & \\
\cline { 2 - 3 } \multicolumn{1}{c}{ Time } & $\begin{array}{c}\text { Number with Order/Total } \\
\text { Number with Urinary Catheter } \\
(\%)\end{array}$ & \\
\hline Weeks 1 to 8 & $7 / 19(37)$ & $17 / 18(94)$ & $<0.001$ \\
Weeks 9 to 16 & $3 / 15(20)$ & $16 / 18(89)$ & $<0.001$ \\
Total & $10 / 34(29)$ & $33 / 36(92)$ & $<0.001$ \\
\hline
\end{tabular}


Table 2. Duration of Urinary Catheterization and Incidence of Urinary Tract Infection by Ward

\begin{tabular}{lcccc}
\hline & $\begin{array}{c}\text { Control Ward } \\
(\mathrm{n}=34)\end{array}$ & $\begin{array}{c}\text { Study Ward } \\
(\mathrm{n}=36)\end{array}$ & $\begin{array}{c}\text { Control-Study Difference } \\
(95 \% \text { Confidence Interval })\end{array}$ & $P$ Value \\
\hline & Number (\%) or Mean \pm SD & & \\
\cline { 2 - 4 } Days of catheterization & $8 \pm 5$ & $5 \pm 3$ & $3(0-5)$ & 0.03 \\
Urinary tract infections & $3(8)$ & $5(14)$ & - & 0.71 \\
\hline
\end{tabular}

restraint" (18). Even when inserted for an appropriate indication, the catheter is often left in place longer than needed (2). The most important risk factor for catheterassociated urinary tract infection is the duration of catheterization $(3,4)$. Because preventing catheter-related urinary tract infection is difficult (19), an intervention that reduces the duration of unneeded catheterization would be beneficial.

Our study had two important findings. First, having a computerized order increased the rate of documentation of indwelling urinary catheter placement from $29 \%$ to $92 \%$. Although documentation alone may not decrease the rates of urinary catheter-related complications, it is important for medical and legal reasons. Second, the mean duration of catheterization was shortened by about one third (3 days) on the study wards. As the study progressed, we discovered that many of the computer-generated alerts were not being acknowledged because of unfamiliarity with the computer system. We believe that the computer-generated reminders would have had an even greater effect if physicians had been more familiar with their use.

The main potential concern about adopting this computerized reminder system is that it could cause premature removal of a urinary catheter. We found no evidence of this, as only 1 of 5 patients who required reinsertion of a catheter had a computer order.

Because most of the resident physicians on the medical wards knew we were conducting this study, we suspect there was some "contamination" of the control ward. Additionally, some catheters were placed when a covering resident from the other ward was responsible for the patient. These factors strengthen our findings, since they would tend to reduce the differences between the study and control wards.

We conclude that using a computer-based order for placing an indwelling urinary catheter in hospitalized patients increased the documentation of urinary catheter placement and decreased the duration of catheterization. We did not have adequate power to detect a difference in the rate of catheter-associated urinary tract infections. While this would be the outcome of most interest, it is strongly correlated with days of urinary catheterization $(3,4,20)$. Future studies may choose to focus on catheterrelated urinary tract infection as the outcome of interest and to assess whether noncomputerized reminders are also effective.

\section{ACKNOWLEDGMENT}

We thank Patricia Hoey, RPh, for computer assistance; Mari Perrotti, RN, and Donna McGrann, RN, for nursing administrative support; Samuel Kaufman, MA, for statistical assistance; and the house staff of the University of Washington, Department of Medicine.

\section{REFERENCES}

1. Haley RW, Hooton TM, Culver DH, et al. Nosocomial infections in U.S. hospitals, 1975-1976; estimated frequency by selected characteristics of patients. Am J Med. 1981;70:947-959.

2. Jain P, Parada JP, David A, Smith LG. Overuse of the indwelling urinary catheter in hospitalized medical patients. Arch Intern Med. 1995;155:1425-1429.

3. Garibaldi RA, Burke JP, Dickman ML, Smith CB. Factors predisposing to bacteriuria during indwelling urethral catheterization. N Engl J Med. 1974;291:215-219.

4. Platt R, Polk BF, Murdock B, Rosner B. Risk factors for nosocomial urinary tract infection. Am J Epidemiol. 1986;124:977-985.

5. Haley RW, Culver DH, White JW, et al. The nationwide nosocomial infection rate. A new need for vital statistics. Am J Epidemiol. 1985;121:159-67.

6. Bryan CS, Reynolds KL. Hospital-acquired bacteremic urinary tract infection: epidemiology and outcome. J Urol. 1984;132:494-497.

7. Saint S, Lipsky BA, Baker PD, et al. Urinary catheters: what type do men and their nurses prefer? J Am Geriatr Soc. 1999;47:1453-1457.

8. Gardam MA, Amihod B, Orenstein P, et al. Overutilization of indwelling urinary catheters and the development of nosocomial urinary tract infections. Clin Perform Qual Health Care. 1998;6:99-102.

9. Saint S, Wiese J, Amory JK, et al. Are physicians aware of which of their patients have indwelling Foley catheters? Am J Med. 2000;109: 476-480.

10. Payne TH. Computer decision support systems. Chest. 2000; 118(suppl):47S-52S.

11. Balas EA, Weingarten S, Garb CT, et al. Improving preventive care by prompting physicians. Arch Intern Med. 2000;160:301-308.

12. Hunt DL, Haynes RB, Hanna SE, et al. Effects of computer-based clinical decision support systems on physician performance and patient outcomes: a systematic review. JAMA. 1998;280:1339-1346.

13. Johnston ME, Langton KB, Haynes RB, et al. Effects of computerbased clinical decision support systems on clinician performance and patient outcome: a critical appraisal of research. Ann Intern Med. 1994;120:135-142.

14. Shea S, DuMouchel W, Bahamonde L. A meta-analysis of 16 randomized controlled trials to evaluate computer-based clinical reminder systems for preventive care in the ambulatory setting. J Am Med Inform Assoc. 1996;3:399-409.

15. McDonald CJ, Hui SL, Smith DM. Reminders to physicians from an introspective computer medical record: a two-year randomized trial. Ann Intern Med. 1985;100:130-138. 
16. Stark RP, Maki DG. Bacteriuria in the catheterized patient. N Engl J Med. 1984;311:560-564.

17. Saint S. State of the science: clinical and economic consequences of nosocomial catheter-related bacteriuria. Am J Infect Control. 2000; 28:68-75.

18. Saint S, Lipsky BA, Goold SD. Indwelling urinary catheters: a onepoint restraint. Ann Intern Med. 2002;137:125-127.

19. Saint S, Lipsky BA. Preventing catheter-related bacteriuria: should we? Can we? How? Arch Intern Med. 1999;159:800-808.

20. Shapiro M, Simchen E, Izraeli S, Sacks TO. A multivariate analysis of risk factors for acquiring bacteriuria in patients with indwelling urinary catheters for longer than 24 hours. Infect Control. 1984;5:525-532.

\section{Alcohol and Medication Interactions in Primary Care Patients: Common and Unrecognized}

Richard Saitz, MD, MPH, Nicholas J. Horton, ScD, Jeffrey H. Samet, MD, MA, MPH

A lcohol use disorders are common and costly (1). Primary care settings are well suited for alcohol screening and intervention. There are valid, brief, practical screening tools; brief interventions can reduce drinking and improve health outcomes; and national practice guidelines recommend these activities $(2,3)$. However, alcohol problems in primary care are often unrecognized and untreated (4).

Interactions between alcohol and medications may be another reason to identify and address alcohol problems in medical settings (5). Physicians are advised routinely to check for drug interactions between medications (6), but it is not known how frequently physicians check for alcohol and medication interactions. We sought to determine the prevalence of potential common alcohol and medication interactions in primary care patients with atrisk, harmful, or dependent (hereafter called "hazardous") drinking, and whether users of medications that interact with alcohol were counseled by physicians about drinking.

\section{METHODS}

The study was approved by the Boston University Medical Center Institutional Review Board. All patients provided written informed consent, and a Certificate of Confidentiality was obtained from the Substance Abuse and Mental Health Services Administration.

English- or Spanish-speaking patients arriving for a visit with one of 41 physicians at an urban academic med- ical center from February 1998 through August 2000 were asked to complete a written alcohol screening test before the physician visit, to assess eligibility for a randomized trial of a systems intervention in primary care (7). Physicians were primary care internal medicine residents and faculty. In that study, half of the physicians received the results of alcohol screening, a preliminary diagnostic assessment, and specific recommendations during a visit. Patients who reported "hazardous drinking," that is, those who had consumed alcohol in the past month and either answered "yes" to at least one of the four "CAGE" alcohol screening questions (modified to refer to the past year rather than lifetime) or who drank at-risk amounts in the past month (8) were eligible for the study. At-risk amounts were defined as a self-report of more than four standard drinks per occasion (three for women) or 14 drinks per week for men (seven for women) in the past 30 days (8).

Subjects were interviewed by a trained staff researcher, both before and after the physician visit. All questions that had not been validated in Spanish were translated, then back-translated and checked for accuracy. Before the visit, subjects were asked whether during the past month they had taken "blood thinners, particularly coumadin or warfarin; antihistamines, such as in allergy pills and cold medicines; opiates or narcotics, such as Percocet or codeine; Motrin, Advil, Aleve, aspirin, ibuprofen or Naprosyn; Tylenol (acetaminophen); sedatives, tranquilizers, medicines to treat anxiety (including benzodiazepines such as Valium and barbiturates such as phenobarbital); antidepressants, medicines to treat depression; or any other medications to help them sleep." We also assessed demographic characteristics, medical and mental health comorbid conditions and symptoms, tobacco use, other drug use, amount of alcohol consumed in the past 30 days, the quality of communication with the physician, and problems due to alcohol (using the Short Inventory of Problems) (9-14).

Immediately after the physician visit, subjects were interviewed to determine if any discussion about alcohol had occurred (15), defined as any discussion about alcohol that included advice or specific counseling about safe drinking limits, advice to cut down or abstain, or referral to an alcohol specialist in a treatment program. All but 3 subjects in the randomized trial (who did not answer questions on the use of medications) were included in this analysis.

\section{Statistical Analysis}

We compared problems due to drinking and other subject characteristics between users and nonusers of these medications with the Fisher's exact test or the Wilcoxon rank sum test, as appropriate. A $P$ value of 0.05 (twosided) indicated statistical significance. 